Ann. Sci. forest., 1982, 39 (4), 439-444

\title{
Le dépérissement du chêne en forêt de Tronçais Les causes écologiques
}

\author{
M. BECKER * et G. LEVY** \\ * I.N.R.A., Laboratoire de Phyto-écologie forestière, \\ ** I.N.R.A., Station de Recherches sur les Sols forestiers, \\ Centre de Recherches forestières de Nancy \\ Champenoux, F 54280 Seichamps
}

\begin{abstract}
Résumé
Seul le chêne pédonculé est victime du dépérissement; le chêne sessile est indemne. L'affaiblissement des arbres remonte à 1976, année de sécheresse exceptionnelle, et les premières mortalités à 1978 . Une étude phyto-écologique montre que les stations sensibles ne correspondent pas du tout à l'écologie connue du chêne pédonculé, dont la présence est soit d'origine artificielle, soit le résultat de la sylviculture passée. Le facteur limitant, dans le cas de la forêt de Tronçais, semble être l'alimentation en eau, tant sur les sols filtrants acides que sur les sols à forte hydromorphie très superficielle. Des facteurs biotiquess secondaires interviennent également et sont en cours d'étude par des spécialistes (champignons pathogènes des racines en particulier). Quelques recommandations sylvicoles sont présentées, ainsi que des propositions de recherches plus approfondies.
\end{abstract}

\section{Introduction}

Un dépérissement inquiétant du chêne s'est développé depuis quelques années dans un certain nombre de régions françaises, à des degrés de gravité variables. Dans le centre du pays, spécialement concerné au vu des résultats d'une enquête nationale de l'O.N.F. en 1980 et 1981, la forêt domaniale de Tronçais apparaît particulièrement touchée.

Ce massif de 10600 hectares est situé dans le nord-ouest du département de l'Allier, à la limite du bocage bourbonnais. Il constitue certainement une des plus prestigieuses futaies de chêne de France.

Les premières observations du phénomène remontent à l'été 1978, avec une accélération très forte à partir de la saison 1979. Le secteur le plus touché se situe dans le bassin du ruisseau de Chandon, plus ou moins centré sur le «Rond de la Cave $\gg: 600$ hectares atteints en mai 1980 , avec 20 p. 100 de chênes morts et 60 p. 100 de dépérissants. La situation s'est encore aggravée au cours des saisons 1980 et 1981 . Ailleurs, le dépérissement est beaucoup plus diffus et concerne des arbres isolés ou des bouquets de surface variable. La majorité des arbres atteints ont entre 80 et 120 ans. 
Un groupe de travail s'est constitué avec des représentants de l'O.N.F. et de diverses disciplines de l'I.N.R.A. (écologie, pathologie, entomologie, télédétection), pour étudier le problème au plan national. Ce sont les premières conclusions, d'ordre écologique, qui sont présentées ici, sur la base des observations faites en forêt de Tronçais.

\section{Résultats des observations}

Les documents établis par Rıom (1981), à la suite de l'interprétation de photographies aériennes faites en 1980 , ont permis d'effectuer un échantillonnage bien adapté des diverses situations, depuis les peuplements parfaitement sains jusqu'aux très dépérissants. Des relevés phyto-écologiques ont été faits (inventaires de végétation et descriptions de sols), complétés par des observations sur l'état du peuplement et par le prélèvement dans les troncs de carottes courtes $(4-6 \mathrm{~cm})$ à $1,30 \mathrm{~m}$ du sol.

La conclusion la plus importante - et dont le caractère absolu n'est pas le moins inattendu - est la suivante : seul le chêne pédonculé est victime du dépérissement.

Malgré sa réputation bien établie de forêt de «rouvres», on savait que le chêne pédonculé existait aussi en proportion notable à Tronçais, mais on ignorait à peu près tout de sa répartition. Son identification certaine nécessite d'ailleurs une bonne expérience et une excellente paire de jumelles...

Quatre types de stations ont été reconnus :

1 - station sur sol non hydromorphe (sinon éventuellement en profondeur) et très acide ;

2 - station sur sol non hydromorphe (sinon éventuellement en profondeur) et mésotrophe ;

3 - station sur sol à forte hydromorphie superficielle et très acide ;

4 - station sur sol à hydromorphie forte mais moins superficielle et moins acide.

Les zones à dépérissement massif sont des zones où le chêne pédonculé est exclusif ou presque ; il est d'ailleurs remarquable de constater que les quelques chênes sessiles présents dans ces conditions sont indemnes. Ces zones correspondent à la station 3, caractérisée par des sols du type pseudogley podzodique et une végétation dominée par Molinia coerulea (L.) Moench. (Molinie), souvent accompagnée par Rhamus frangula L. (Bourdaine) ; les autres espèces acidiphiles sont également présentes. On se trouve en général en situation topographique basse, mais peu accusée.

Dans les zones à dépérissement moins spectaculaire, voire diffus, l'intensité du phénomène apparaît liée essentiellement, tout banalement, au pourcentage de chêne pédonculé dans le peuplement...

Les stations appartiennent alors, soit au type précédent - mais représenté sur de faibles surfaces seulement, très dépérissantes -, soit aux types 1,2 et 4.

Si l'on tente d'apprécier la santé du chêne pédonculé sans préjuger de sa représentation dans le peuplement, il est cependant possible de classer comme suit les 
stations, par sensibilité décroissante au dépérissement : station $3>$ station $1>$ station $4>$ station 2 .

Le type 1 est caractérisé par un sol brun acide à ocre podzolique (souvent marmorisé en profondeur), très acide et sujet à se déssecher fortement, à végétation herbacée nettement acidiphile : Calluna vulgaris (L.) Hull (Callune), Pteridium aquilinum (L.) Kuhn. (Fougère aigle), Deschampsia flexuosa (L.) Trin. (Canche flexueuse), Polytrichum formosum Hedw. (Polytric élégant)...

Le type 2 est caractérisé par un sol brun mésotrophe ou brun lessivé (souvent marmorisé en profondeur), également filtrant et sujet à se dessécher, mais sans doute moins que dans le type précédent, en raison d'une texture moins grossière en profondeur ou d'une situation topographique de bas de pente. La végétation herbacée est neutro-acidophile : Rubus sp. (Ronce), Polystichum filix mas (L.) Roth. (Fougère mâle), Athyrium filix femina (L.) Roth. (Fougère femelle), Atrichum undulatum P. Beauv. (Mousse), Melica uniflora Retz (Mélique à une fleur), Euphorbia amygdaloides L. (Euphorbe des bois), Brachypodium silvaticum (Huds.) R. et S. (Brachypode des bois)...

Le type 4 est peu représenté. Le sol se présente comme un sol brun lessivé à pseudogley. Selon les cas, la végétation est nettement acidiphile (moins cependant que dans les stations 1 et 3 ) ou à tendance neutro-acidophile ; la Molinie peut être présente, mais sans jamais former de tapis denses.

Les observations faites sur les mini-carottes prélevées dans les troncs montrent de façon irréfutable que le début du processus de dépérissement remonte à 1976, année de sécheresse exceptionnelle, suivant elle-même une année à déficit hydrique marqué.

Chez le chêne sessile, on n'observe qu'un léger fléchissement de l'accroissement en 1976 et 1977, suivi d'une reprise tout à fait normale. Chez le pédonculé, le fléchissement de 1976, et surtout de 1977, est en moyenne beaucoup plus marqué; ensuite plusieurs cas de figures sont possibles : reprise plus ou moins rapide, stagnation, ou accentuation du fléchissement allant jusqu'à des cernes indiscernables à l'œil. Il apparaît en tout cas que l'observation de ces mini-carottes fournit de bien meilleures informations sur l'état de santé réel d'un arbre que celle de l'aspect actuel du houppier, qui est apparu parfois trompeur.

En outre, toutes choses égales par ailleurs (diamètre et station), l'aubier du chêne pédonculé apparaît plus mince $(-35$ p. 100) que celui du sessile : ce caractère, s'il est favorable sur le plan de l'utilisation du bois, pourrait contribuer à expliquer la plus grande sensibilité du pédonculé à la sécheresse (capacité de stockage de l'eau moindre).

\section{Causes probables du dépérissement}

Dans l'état actue1 des connaissances, qui nécessiteraient d'ailleurs des vérifications expérimentales - urgentes au vu des implications phytosanitaires en cause -, les deux espèces de chêne, dans leurs formes bien typées, sont caractérisées par les écologies suivantes:

- Chêne pédonculé : espèce assez exigeante sur le ñlan de la richesse minérale du sol, tolérant bien l'hydromorphie temporaire, résistant mal à la sécheresse. 
- Chêne sessile : espèce nettement plus rustique, s’accommodant de sols plus pauvres, tolérant assez bien l'hydromorphie, résistant beaucoup mieux à la sécheresse.

Les sols de Tronçais, surtout ceux des stations 3 et 1 , apparaissent done nettement défavorables au chêne pédonculé : d'une part par leur pauvreté chimique (surtout en calcium, $\mathrm{Ca}^{++}$), d'autre part, et surtout, par leur régime hydrique. Les sols brun acide à ocre podzolique observés, en raison de leur texture, ont une faible capacité de rétention en eau, que peut ne pas compenser, surtout dans les parties hautes du relief, leur profondeur utile souvent satisfaisante. Quant aux sols hydromorphes, la présence d'une nappe crée des conditions défavorables à l'enracinement, même pour une espèce relativement résistante à l'engorgement comme le chêne pédonculé ; le volume de sol prospecté est donc moindre, ce qui rend les arbres plus sensibles à la sécheresse, sensibilité qui est encore accentuée par la texture sableuse des horizons supérieurs. Ceci explique ce paradoxe apparent, à savoir que le chêne pédonculé soit tout spécialement exposé aux risques de sécheresse sur les sols très hydromorphes de la station 3.

La sécheresse de 1976 a donc été à l'origine du dépérissement du chêne pédonculé en raison de la faible réserve en eau utilisable de la plupart des sols de Tronçais. Ses conséquences ont été beaucoup moins catastrophiques dans les stations où cette réserve est plus importante du fait, soit d'une texture un peu moins grossière en profondeur ou d'une situation topographique plus favorable dans le cas des sols filtrants (station 2), soit d'une hydromorphie moins superficielle (station 4).

Il est possible que la moins grande pauvreté chimique des sols des stations 2 et 4 , qui se traduit en particulier dans l'horizon $\mathrm{A} 1$ par un $\mathrm{pH}$ et un taux de saturation $\mathrm{S} / \mathrm{T}$ plus élevés, et par un rapport $\mathrm{C} / \mathrm{N}$ plus bas, constitue également un élément favorable dans la résistance au dépérissement.

\section{Enchaînement probable du processus}

On peut s'étonner de l'abondance du chêne pédonculé dans les stations qui semblent correspondre si peu à son écologie. Mais on sait que des reboisements importants ont eu lieu à Tronçais au siècle dernier, par semis et plantations. Il est hautement probable que l'on ait alors récolté à cette fin des glands là où ils étaient les plus abondants, c'est-à-dire sur des arbres de «plaine» ou de lisière, qui sont le plus souvent des chênes pédonculés...

Ces glands auraient surtout servi à reboiser les zones hydromorphes, peut-être depuis déjà assez longtemps passées à l'état de landes (ainsi que semble d'ailleurs en témoigner la toponymie locale), et en tout cas difficiles à régénérer naturellement. Ils auraient pu également servir çà et là en forêt, sur des surfaces variables, pour des compléments de régénération.

Les recherches historiques de Roy (1977) montrent que des semis de glands ont également eu lieu dans un passé plus lointain, aux XVII ${ }^{\mathrm{e}}$ et $\mathrm{XVIII}^{\mathrm{e}}$ siècles.

A cette artificialisation délibérée, qui a d'ailleurs affecté beaucoup d'autres forêts, a pu aussi s'ajouter un phénomène spontané, donc plus insidieux, et de portée également générale pour la forêt française. Etant donné la longueur des générations, cette forêt est encore une copie assez peu déformée — au moins dans sa composition spécifique - 
de celle d'il y a plusieurs siècles. Or, l'on sait que celle-ci était souvent dans un état de surexploitation et de dégradation très avancée. Pour diverses raisons, et en particulier à cause de son caractère plus héliophile, le chêne pédonculé aurait alors pu "sortir » de ses stations naturelles et remplacer le hêtre et le chêne sessile dans les forêts clairsemées. Cette hypothèse était déjà émise par TuRc dès 1927, à la suite d'un dépérissement semblable sur le plateau nivernais. Quoi qu'il en soit, ces chênes pédonculés ont le plus souvent donné des arbres de qualité apparemment comparable à celle des chênes sessiles, et il aura fallu attendre l'accident climatique constitué par les sécheresses de 1975 et, surtout, de 1976 pour que s'exprime le verdict écologique.

Toutefois, cette sorte de sanction, qui condamne ainsi de façon aussi brutale et sélective des peuplements centenaires, nous paraît trop absolue pour ne faire intervenir que des facteurs abiotiques. Des études sont en cours pour préciser la part qu'ont pu, et que peuvent encore, jouer d'autres facteurs, biotiques ceux-là, pour aggraver ou précipiter le phénomène : facteurs entomologiques (chenilles défoliatrices en particulier) et surtout sans doute facteurs pathologiques (Ö̈dium, Armillaire et d'autres champignons, non encore identifiés à ce jour, se développant sur les racines).

\section{Conclusions provisoires}

Pour la forêt de Tronçais, la conclusion pratique principale est plutôt optimiste, en ce sens que le dépérissement devrait s'y limiter, au pire, à la disparition du chêne pédonculé. Elle deviendrait alors effectivement la grande forêt de «rouvres» tant vantée...

En ce qui concerne la conduite à tenir dans l'immédiat, diverses suggestions peuvent être faites:

- prêter une attention très précise à la nature des espèces de chêne en présence, en particulier au moment des coupes de régénération, pour ne laisser se régénérer que le sessile, quitte à faire des compléments plus ou moins larges par plantation (en exigeant alors du producteur de plants la livraison effective de chêne sessile...) ;

- précipiter, dans la mesure du possible, l'extraction des chênes pédonculés sans attendre une nouvelle vague de dépérissement ; cette attitude pourrait avoir le mérite d'une part de limiter les risques de voir se déclencher (ou s'aggraver?) un processus épidémique, dont l'éventualité n'est toujours pas écartée, susceptible de gagner les chênes sessiles encore apparemment réfractaires, d'autre part de supprimer, s'il en est encore temps, les risques de «pollution génétique », c'est-à-dire d'abâtardissement des futures générations de chênaies sessiles par le pollen des chênes pédonculés avoisinants ;

- ou, au contraire, intervenir pour tenter de sauver les peuplement de chênes pédonculés encore indemnes ou ne présentant que des signes limités de dépérissement ; des expériences de fertilisation sont en cours dans cette optique.

Il n’est guère possible encore de fournir au gestionnaire des arguments décisifs en faveur de l'une ou l'autre de ces deux dernières attitudes, pourtant opposées. Pour les peuplements âgés et fertiles, notre préférence irait plutôt, étant donné l'urgence, à l'avant-dernière (élimination du pédonculé). Pour les autres peuplements, il devrait être possible d'attendre les conclusions des recherches en cours ou à venir. D'ores et 
déjà, il convient d'avoir à l'esprit l'impact négatif prévisible de la fertilisation sur la largeur des cernes, et donc sur la qualité du bois. Par ailleurs, nous pouvons également formellement déconseiller le drainage des stations très hydromorphes lorsque les peuplements sont déjà adultes (BECKER, LÉvY, à paraître).

Enfin, un certain nombre de recherches sont à entreprendre ou à approfondir :

- en premier lieu sur la taxinomie de nos chênes à feuilles caduques, sur la mise au point de critères d'identification plus sûrs et sur l'irritante question des hybridations (réalité de ces phénomènes, fréquence, variabilité infraspécifique) ;

- sur l'écophysiologie respective des diverses espèces (chêne pédonculé et chêne sessile essentiellement), en particulier sur leur comportement hydrique (résistance à la sécheresse, à l'engorgement, aux alternances d'excès et de déficit en eau), en interaction avec leur nutrition minérale sur les divers types de substrats;

- sur le statut du chêne pédonculé dans la forêt française ; il serait ainsi souhaitable de déterminer, dans les diverses régions, les stations dans lesquelles cette essence apparaît ne pas être à sa place sur le plan écologique, et où elle est donc moins armée pour résister à un accident climatique, surtout lorsque viennent s'y ajouter des attaques d'origine fongique ou entomologique.

Reçu pour publication en juillet 1982.

\section{Summary}

Oak wilt disease in the forest of Tronçais (France). Ecological causes

Pedunculate oak only (Quercus robur L.) is suffering this disease ; sessile oak (Quercus petraea (M.) Liebl.) is uninjured. The weakening of the trees began in 1976, year characterized by an exceptional drought, and the first mortalities in 1978. A plant ecological study shows that the damaged sites don't suit the known ecology of pedunculate oak, which presence either has an artificial origin, or is the result of the past silviculture. In the forest of Tronçais the most important ecological factor seems to be the water supply, both on the pervious acid soils and on the soils which are characterized by an important and superficial waterlogging. Secondary biotic factors occur too, which are studied at present by specialists (chiefly root pathogenic fungi). Some silvicultural recommendations and propositions for more extensive researches are proposed.

\section{Références bibliographiques}

BeCKer M., LÉvy G. Les causes écologiques du dépérissement du chêne en forêt de Tronçais (Allier). Premières conclusions. Rev. for. fr., à paraître.

Rıom J., 1981. Le dépérissement du chêne en forêt de Tronçais. Etude par photographies aériennes. Document interne, I.N.R.A. Télédétection.

Roy F.X., 1977. La forêt domaniale de Tronçais. Rev. for. fr., 29, $\mathrm{n}^{\circ}$ spécial (Eléments d'histoire forestière), 82-89.

Turc L., 1927. Note sur le dépérissement du chêne pédonculé dans les forêts du plateau nivernais. Rev. Eaux Forêts, 65, 11, 561-565. 